\title{
A MULTICHANNEL THOMSON SCATTERING APPARATUS
}

EY

N. BRETZ, D. DIMOCK, V. FOOTE,

D. JOHNSON, D. LONG,

AND

E. TOLNAS

PLASMA PHYSICS
LABORATORY

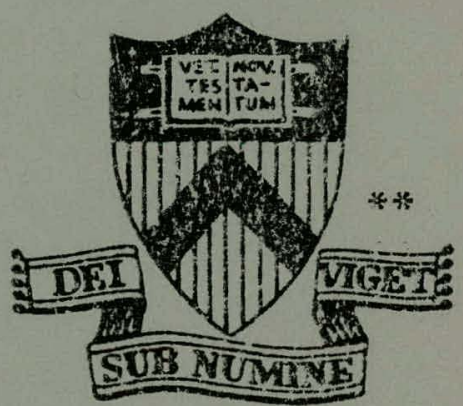

DISTREUTHON OF THIS DOCUMENT IS UNLIMITED

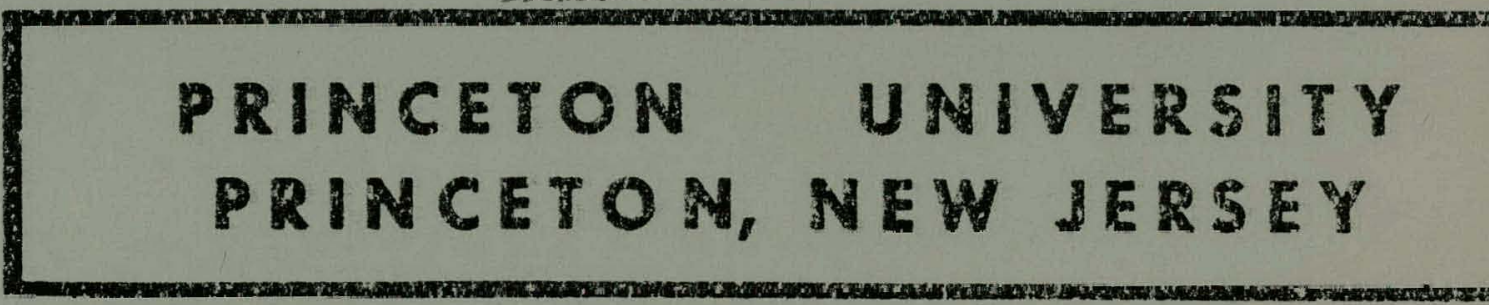

This work was supported by U. S. Energy Research and Developrent Administration Contract EX-76-C-C2-3073. Reproduction, transiation, publication, use and disposal, in whole or in part, by or for the United States Govermint is permitter. 


\section{DISCLAIMER}

This report was prepared as an account of work sponsored by an agency of the United States Government. Neither the United States Government nor any agency Thereof, nor any of their employees, makes any warranty, express or implied, or assumes any legal liability or responsibility for the accuracy, completeness, or usefulness of any information, apparatus, product, or process disclosed, or represents that its use would not infringe privately owned rights. Reference herein to any specific commercial product, process, or service by trade name, trademark, manufacturer, or otherwise does not necessarily constitute or imply its endorsement, recommendation, or favoring by the United States Government or any agency thereof. The views and opinions of authors expressed herein do not necessarily state or reflect those of the United States Government or any agency thereof. 


\section{DISCLAIMER}

Portions of this document may be illegible in electronic image products. Images are produced from the best available original document. 


\section{NOTICE}

This report was prepared as an account of work sponsored by the United States Government. Neither the United States nor the United States Energy Research and Development Administration, nor any of their employees, nor any of their contractors, subcontractors, or their employees, makes any warranty, express or implied, or assumes any legal liability or responsibility for the accuracy, completeness or usefulness of any information, apparatus, product or process disclosed, or represents that its use would not infringe privately owned Iights.

Printed in the United States of America.

Available from

National Technical Information Service

U. S. Department of Commerce 5285 Port Royal Road

Springfield, Virginia 22151

Price: Printed Copy $\$ \ldots$; Microfiche $\$ 3.00$

*Pages
$1-50$
$51-150$
$151-325$
$326-500$
$501-1000$

NTIS

Selling Price

$\$ 4.00$

5.45

7.60

10.60

13.60 


\title{
A MULTICHANNEL THOMSON SCATTERING APPARATUS
}

\author{
N. Bretz, D. Dimock, V. Foote, D. Johnson, \\ D. Long, and E. Tolnas \\ Plasma Physics Laboratory, Princeton University \\ Princeton, New Jersey 08540
}

PPPL-1356

July 1977

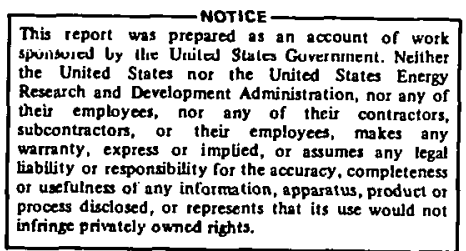

process disclosed, or represents that its use would not 
A MULTICHANNEL THOMSON SCATTERING APPARATUS *

N. BRETZ, D. DIMOCK, V. FOOTE, D. JOHNSON, D. LONG', and E. TOLNAS

Plasma Physics Laboratory, Princeton University

Princeton, New Jersey 08540

\section{ABSTRACT}

A Thomson scattering apparatus for measuring the electron temperature and density along a $90 \mathrm{~cm}$ diameter of the PLT plasma has been built. A wide angle objective images the $3 \mathrm{~mm} \times 900 \mathrm{~mm}$ ruby laser beam onto an image dissector which rearranges the 300:1 image to 20:1 forming the input slit of a spectrometer. The stigmatic spectrometer provides 20 wavelength elements of $\sim 70 \mathrm{~A}$ each. A micro-channel-plate image itensifier optically coupled to a cooled SIT tube provides detection with single frame linearity and 1000:I dynamic range. Spatial profiles of $\mathrm{N}_{\mathrm{e}}$ and $\mathrm{T}_{\mathrm{e}}$ in the range $10^{13}-10^{14} \mathrm{~cm}^{\cdots 3}$ and $0.05-3 \mathrm{keV}$ have an accuracy of $30 \sqrt{10^{13} / \mathrm{N}_{\mathrm{e}}\left(\mathrm{cm}^{-3}\right)}$ \% per $1.2 \mathrm{~cm}$ element. 
Introduction

With the development of larger and more complicated plasma -generators for fusion studies there has been a growth in the data gathering capacity of instruments which measure various plasma parameters. We shall describe an expansion of a relatively standard method for measuring the electron temperature and density - Thomson scattering. Most instruments based on this principle have yielded a value for $\mathrm{N}_{e}$ and $\mathrm{T}_{\mathrm{e}}$ in a single volume element at a single time.(1). The evolution of a plasma discharge could only be mapped out by making repeated measurements at different times and in different locations. In tokamak devices this state of affairs has been determined by the electron density range $\left(10^{13} .-10^{14} \mathrm{~cm}^{-3}\right)$ for which an intense pulsed laser source is required in order to obtain adequate statistics. The Thomson scattering apparatus currently in use on the PLT (Princeton Large Torus) tokamak can make simultaneous measurements at a large number of positions alonq a vertical chord thrọgh the plasma by using a narrow beam and collecting light along the entire length. The resulting temperature and density profiles are used to estimate such important parameters as the electron energy confinement time, the average effective charge of ions, and the energy content of the plasma (2). Since this information can acquired between machine cycles (2-10 minutes) the time required to tune the discharge to a given condition or to characterize the evolution of a discharge has been considerably reduced. The temporal evolution must still be acquired by repeating the discharge and measuring at different times.

The apparatus consists of two parts; a pulsed ruby laser light source which passes a narrow beam through the plasma and a stigmatic 
spectrometer which spatially and spectrally resolves the light scattered by the plasma. The light from the laser passes vertically along the $90 \mathrm{~cm}$ minor diameter of the torus. The image of the laser beam is focused by a wide angle lens onto an array of light pipes which reform the image as the entrance slit of a spectrometer. The device resolves 76 spatial channels, 20 of which are for measurement of the plasma background intensity, and 20 wavelength intervals chosen to allow temperature measurements in the range $50 \mathrm{eV}$ to $5 \mathrm{keV}$. The limited access to the tokamak and the high optical throughput required of the system create a number of mechanical, optical, and electro-optical problems which will be discussed. The optics are designed to match a very narrow image onto a circular photocathode while maximizing the amount of light collected. The boundary conditions are the width of the narrowest beam which can practically be transmitted through the plasma and the diameter of the largest suitable detector ( $4 \mathrm{~cm}$ ). The amount of light which can be collected is ultimately limited by the area of the detector. For a given detector size and imaging at $f / l$, a larger cone of light can be collected if the area, and therefore the width, of the source is as small as possible. Minimizing the source width has the additional advantage that less plasma light will be collected. We have assembled a ruby laser which produces 2-4 Joules of energy with a beam divergence such that $85 \%$ of the energy falls within $0.25 \mathrm{mrad}$. The beam is focused over a $6 \mathrm{~m}$. path on the center of the vacuum vessel, so that the beam is no wider than $2 \mathrm{~mm}$ in the plasma. However, in order to allow for some alignment. error we collect light from a $3 \mathrm{~mm} \times 900 \mathrm{~mm}$ slot. The alignment of the laser beam with respect to the spectrometer is 
critical and must be maintained while the $35 \mathrm{kG}$ toroidal field and $700 \mathrm{kA}$ Ohmic heating pulse cáuse the 150 ton machine to oscillate perceptibly.

The light collection optics; spectrometer, and detector are integrated into a single structure. The objective lens forms an image of the laser beam on a set of fiber optic light pipes. The area transformation carried out by the fibers changes the $300: 1$ aspect ratio of the slot to $14: 1$ while retaining 56 elements of spatial resolution. 'l'he objective also forms an image of a slot parallel to the laser beam which is used to monitor the plasma background light. This image is sampled with less spatial resolution than the scattered light, area transformed by the light pipes, and stacked above and below the laser beam image to make an input slit for the spectrometer which has an aspect ratio of $20: 1$. The central portion of the slit contains the scattered light and the top and bottom parts are a split field containing background plasma light. The 20:1 input slit and our requirement for 20 wavelength intervals to span the temperature range of the plasma are compatible with a square output field in the spectrometer. This field is reimaged onto the photocathode of an image intensifier. Thus the fiber optic area transformation is necessary to efficiently use the circular detector area. To further simplify the image analysis the spectrometer has been designed so that the output field is an approximately cartesian grid with spatial information vertical (along the slit) and wavelength dispersed in the horizontal direction. Finally we are limited by the best optics to imaging at $f / l . l$ onto the detector, so we find that a $28 \mathrm{~mm}$ x $28 \mathrm{~mm}$ usable photocathode area translates to an input cone of about $f / 9$ for the objectıve lens. 
The detector consists of a micro-channel-plate (MCP) image intensifier fiber optically coupled to a silicon-intensified-target (SIT) television tube. The first photocathode is shuttered with a 100 ns electrical gate which is synchronized with the laser pulse. The intensified light from the phosphor of the MCP intensifier is gated through the SIT tube and integrated on the target. The target is cooled to decrease the leakage which increases the dynamic range and allows the target to be used as a storage medium during the electrically noisy period when the tokamak is operating and to be read out several seconds later. The camera has a shutter ratıo of about $10^{8}$, a linear dynamic range of $1000: 1$, and a sensitivity limited by shot noise from the MCP photocathode. In developing the camera we have drawn on the experience of several astronomical groups who have had considerable experience in sillıcon vidicon photometry $(3,4,5)$. In order to calculate $\mathrm{N}_{e}$ and $\mathrm{T}_{e}$ from the raw data we need to subtract off background plasma light, stray light from the laser which makes its way undispersed through the spectrometer, and the dark current level from the target. In addition we need to have a file of the sensitivity and noise factors for each picture element. Using this information we fit the scattered spectrum to a nearly Gaussian line shape for each of our position elements. The temperature is obtained from the width and the density from the area of the fitted curve. The data management and fitting calculations are handled as part of the PLT data acquisition system which consists of a PDP-10 computer interfaced via PDP - 11/40's to various data sources. 
Apparatus Overview

Figure 1 shows the relationship between the laser, tokamak, and spectrometer. The laser rests on a stationary mount on the basement floor. Its beam travels parallel to the same major radius of the torus along which we observe the scattered light. The beam is directed vertically toward the plasma by a $45^{\circ}$ prism and focusing element which may be positioned beneath any one of 10 beam lines. The prism and s̈pectrometer may be translated together to scan the horizontal plasma dimension shot-by-shot. To reduce the probability of laser induced damage the vacuum vessel windows have been put near the beam deflector at the end of long pipes. The beam dumps are similarly located at the ends of evacuated pipes extending above the plasma. The spectrometer views the laser beam through a $25 \mathrm{~cm}$. diameter fused quartz window and can be rolled in and out on a track to scan the vertical beam paths or to allow maintenance on PLT.

The viewing access is restricted above and below the vacuum window by coils in the Ohmic heating primary system and further away from the vessel by a massive torque frame which holds the toroidal field coils in place. The objective lens is mounted on a. rigid phenolic snout that protrudes in through these obstructions. The light is relayed by the image dissector and transfer lens to a box which houses the spectrometer and television camera. The fringing field from the plasma and interior coil structure (not shown) are about $300 \mathrm{G}$ with $\frac{\mathrm{dB}}{\mathrm{dt}} \leq 5 \times 10^{4} \mathrm{G} / \mathrm{s}$. at the camera. The entire apparatus must be operated remotely because of the dangers of high pulsed fields and currents, because of an erratic hard X-ray flux, and (hopefully) because of a high flux of thermal neutrons. 
Laser

The main feature of the laser is its low divergence; $85 \%$ of the energy falls within a $0.25 \mathrm{mrad}$. cone. Otherwise the energy, 2-4 Joules, and output beam diameter of $1.9 \mathrm{~cm}$ are similar to that of other scattering systems with which we have had considerable experience. The line width is typically less than $1 \stackrel{\circ}{\mathrm{A}}$ and the pulse width about $20 \mathrm{~ns}$. The laser system is shown in figure 2 . To achieve the low divergence one begins with an oscillator constrained to operate in a TEM ${ }_{\text {Oo }}$ mode by a small cavity aperture. Near diffraction limited performance can be achieved with a high quality rod. Our system is built of Korad components. The oscillator is a $\mathrm{Kl}$ head which consists of a $1.0 \mathrm{~cm}$ diameter $\mathrm{x} 10 \mathrm{~cm}$ long ruby with a $1.7 \mathrm{~mm}$ diameter aperture. The $50 \mathrm{~cm}$ cavity is plane-parallel with a Pockel's cell Q-switch (Korad KQ 52). The oscillator produces about $100 \mathrm{~mJ}$ which is amplified in two stages. Following the oscillator is a negative lens which causes the beam to diverge slowly through the amplifiers so that the energy density is roughly constant. The amplıfiers are a Korad $\mathrm{k} 6(6 \mathrm{~mm}$ diameter $\mathrm{x} 20 \mathrm{~cm}$ ruby)and a $\mathrm{K} 5$ (19 mm diameter $\times 15$ cm ruby) each of which use selected high quality rods. With this energy density the expected life of the rods is about 10,000 shots. However, the data on whlch our lifetime estimates are made is sparse $(6,7)$. We have experienced our only rod damage after about 7,000 shots (to the output face of the last amplifier) however no deterioration in the divergence was observed prior to this. The output beam is made parallel with a simple positive lens. The beam divergence was measured by focusing a fraction of the beam over a long path and measuring the energy passing through a set of apertures. These measurements showed that the main aberration 
in the beam was astigmatism oriented along the principle axes of the ruby. This aberration was corrected optically for the stated divergence; however, it is left uncorrected in the scattering system and the beam focused so that its width in the scattering plane is slightly larger than its width as viewed from the objective lens. Divergence measurements using photographic techniques ( 8 ) were generally found to be misleading. 


\section{Beam Optics}

The laser beam is directed up through the torus and focused on the plasma center by a $45^{\circ}$ prism and lens which ride on a small granite sled. The sled can be pulled across a precision ground flat granite table by a lead screw in order to select one of the vertical beam lines. The mechanical stability and reproducability of the table setting allow an accuracy of about 0.02 mrad in the beam direction. Some systemmatic distortions in the table surface are corrected by selectively orienting the wedge in the vacuum windows.

The tubes which carry the beam to the torus have apertures placed at regular intervals to cut down wall reflections. The tube diameter is tapered in several segments from $2.5 \mathrm{~cm}$ at the window to $1.5 \mathrm{~cm}$ near the vacuum vessel where space is limited by poloidal field coils and support structure. The beam dumps are located about $2 \mathrm{~m}$. above the plasma midplane and consist of flat tungsten surfaces slanted $10^{\circ}$ away from the direction of the beam. Our rationale is that tungsten will be damaged least by the beam and thus be less likely to throw impurities into the plasma. In any case the power density at the dump $\left(\sim 5 \times 10^{8} \mathrm{w} / \mathrm{cm}^{2}\right)$ is high enough that the backscattered intensity is relatively insensitive to the dump geometry or material.

When the apertures are properly aligned concentric to the beam the stray laser light which is collected by the spectrometer comes mainly from the dump. In order to trap this light we have put a semicircular "viewing" dump inside the vacuum chamber. Figure 3 is a wide angle photograph of this dump as seen through the scattering window. It consists of a stack of (non-magnetic) razor 
blades which extends around the inside surface of torus. The dump is especially important near the beam entry and exit in trapping the collimated light from the beam dump. 


\section{Light Collection Optics and Spectrometer}

The scattered spectrum has a width of about $\left.850: \mathrm{FT}_{\mathrm{e}}(\mathrm{KeV})\right]^{1 / 2} \AA$ (FWHM at $\theta_{S}=90^{\circ}$ ) centered about the $6943 \AA$ laser line. However because the quantum efficiency of our photocathode is falling off sharply beyond $7000 \AA$ we will be concerned only with the spectral band $5500 \AA$ A $-6900 \AA$. The light collection optics and spectrometer have been optimized for this range.

There are several unusual requirements of the optical design. First the viewing field of the objective lens has to be about $60^{\circ}$ in order to see the top and bottom edges of the plasma and we will be collecting light at $\mathrm{f} / 8$. Second the fiber optic image dissection must be accomplished efficiently and without significantly spreading the solid angle with which light enters the fibers. Finally the spectrometer has to resolve 80 elements of information along the slit and should reduce the grating curvature in the output field to a few percent while accepting light at $f / 4.5$. The overall

design aims to maximize total transmission by using anti-reflection coated optics and a minimum number of surfaces. We will describe the optical train shown in Figure 4 element by element starting with the objective.

The wide angle objective is a concentric anchromat (9). It is nominally a $25 \mathrm{~cm}$ focal length, $\mathrm{f} / 2$ lens which is used to magnify the image by 0.53 . The lens is a simple doublet using common glass types. When apertured at $f / 3$ the composite lateral aberrations are less than $0.3 \mathrm{~mm}$ and the transmission is $90 \%$. While the aberrations in the lens are for our purposes negligible the image field is curved. The elements of the image dissector are clamped along the curved image of the beam with the fiber bundle surfaces normal to the 
central rays.: The dissector and clamp are shown in figure 5 . There are 56 elements $1.6 \mathrm{~mm}$ wide by $6 \div 3 \mathrm{~mm}$ long evenly spaced along the image of the laser beam. On a parallel arc there are 20 identical elements which sample the plasma light. We assume that the plasma light in the scattering volume will be nearly the same; we expect that the spatial variation in the plasma light will be small and that it's intensity will typically be less than $2 \%$ of the scattered light. Each fiber bundle is rotated $90^{\circ}$ and repacked; the scattering channels occupy the center of the re-tormed sint, and half the background channels are above and half below the main data channels. With allowances for spaces between signal and plasma light channels the new image is $6.3 \mathrm{~mm}$ wide by $125 \mathrm{~mm}$ high. This image is transferred 1:1 by a relay lens onto the input slit of the spectrometer. The elements of the image dissector were selected after testing fiber optics from several manufacturers and some homemade lucite strips. In order to maintain a high transmission for light within an approximately $\mathrm{f} / 4$ cone, the fibers must have a high core to cladding area, uniform packing, and low internal loss. At the moment our best solution consists of bundles of $75 \mu \mathrm{m}$ diameter clad glass fibers manufactured by the Fiber "Photics Division of Skia Corp. On testing over 100 bundles $45 \mathrm{~cm}$ long, filling at $\mathrm{f} / 4$ and extracting at $\mathrm{f} / 4.5$, we find an average transmission of $35 \%$.

The spectrometer uses a Littrow mount in first order and has a nominally $45 \mathrm{~cm}$ focal length and $f / 4.5$ aperature. We take full advantage of the flexibility allowed by the fiber optic inputimage by arranging the output ends of the elements on a curved focal plane (to reduce the output field curature) and curving the slit (to minimize the distortion in the output field caused by the planar grating). Figure 6 shows schematically the input slit and 
output field of the instrument. The bending of the input slit produces a straight vertical image only at the center of the output field (10). At the largest and smallest wavelengths the vertical linear distortion is less than 1\%. The plano-convex Littrow lens is made of a high index (Lakl0) glass oriented to reduce spherical aberrations (the usual solution of using a doublet introduces unacceptable reflections). The aberrations in the spectrometer together with its input optics were optimized using a ray tracing program. The $10 \mathrm{~cm} \times 15 \mathrm{~cm}$ grating has $1800 \mathrm{l} / \mathrm{mm}$ and is blazed In first order at $6200 \AA$. The transmission of the grating and Littrow lens is $50 \%$ for unpolarized light.

A camera lens reduces the output field of the spectrometer 4.5:1 onto the MCP photocathode. This lens was designed and manufactured for us by Diffraction Optics Inc. It has an aperture of F/0.9 with $80 \%$ transmission and an MTF $\simeq 0.5$ at $5 \mathrm{l} / \mathrm{mm}$. Between the camera lens and the spectrometer output field we have inserted a wedge to reduce chromatic aberrations from the simple Littrow lens. Distortions in the spectrometer field, camera lens, and TV camera can be matched by proper design and placement of the field lens. The transmission from the laser beam to the MCP photocathode is about $7 \%$.

Our overall resolution has only been measured through the TV system. If a single scattering channel is illuminated we observe that most of the signal appears on the TV scan line associated with that channel and $20 \%$ of that signal appears on the two adjacent scan lines. The situation is somewhat worse on the top and bottom of the field where the plasma background is dispersed. There distortion causes a mismatch between the image and the TV scan line so that at the corners of the field the illuminated and next lower line have about equal signal. The imperfect resolution noted here will 
restrict the largest temperature and density gradients which can be observed.

A number of peripheral features have been incorporated into the spectrometer to take advantage of the information capacity of the TV detector. In regions of the field where the data is expected to be relatively unimportant, light pipes are mounted which serve as monitors for the laser beam intensity, the 6943 A stray light in the spectrometer, and misalignment of the beam with respect to lile input silt. we have also blocked out the $H_{\alpha}$ line so that no part of the detector is saturated in normal operation.

Detector

Our objective is to have enough sensitivity to measure electron temperatures and densities in the range $50 \mathrm{eV}-5 \mathrm{keV}$ and $10^{13}-10^{14} \mathrm{~cm}^{-3}$ with an accuracy better than $10 \%$. This requixed a detector whose sensitivity is limited by shot noise from the best red sensitive photocathodes. We estimate the number of photoelectrons: per pixel (unit of TV resolutions) scattered by the plasma to be

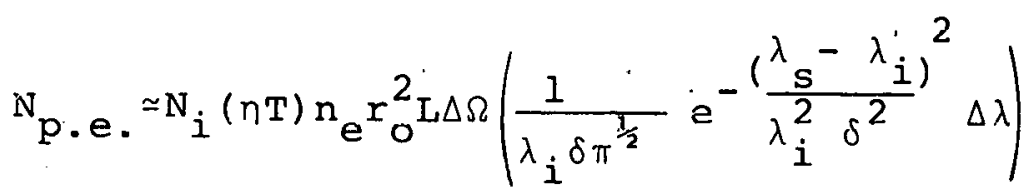

where

$$
\begin{aligned}
\mathrm{N}_{i} & =\text { number of incident photons }\left(\sim 10^{19}-3 \mathrm{~J}\right) \\
n & =\text { photocathode quantum efficiency }(\sim 0.0 \mathrm{~J}) \\
\mathrm{T} & =\text { transmission of optical train }(\sim 0.07) \\
\mathrm{n}_{\mathrm{e}} & =\text { electron density } \\
\mathrm{r}_{0} & =\text { classicial electron radius }\left(2.8 \times 10^{-13} \mathrm{~cm}\right) \\
\mathrm{L} & =\text { laser beam length sampled by pixel }(\sim 1.2 \mathrm{~cm}) \\
\Delta \Omega & =\text { collection solid angle }\left(\sim 10^{-2}\right) \\
\lambda_{\mathrm{S}, i} & =\text { scattered, incident wavelength }
\end{aligned}
$$




$$
\begin{aligned}
\Delta \lambda & =\text { wavelength interval sampled by one pixel }(\sim 70 \AA \text { ) } \\
\delta & =\left[\frac{8 \mathrm{KT}}{\mathrm{mc}^{2}}\right]^{1 / 2} \sin \theta_{s} / 2 \\
\theta_{s} & =\text { angle between incident and scattered waves }\left(\sim 90^{\circ}\right)
\end{aligned}
$$

If we take the nominal set of values indicated in parentheses for $\mathrm{N}_{\mathrm{e}} \simeq 10^{13} \mathrm{~cm}^{-3}, \mathrm{~T}_{\mathrm{e}}=1 \mathrm{keV}$ we have

$$
\begin{aligned}
& \text { pixel or } \mathrm{N}_{\mathrm{p} . \mathrm{e} .} \simeq 30 e^{-\left(\lambda_{s}-\lambda_{i}\right)^{2} /(614 \AA)^{2}} \text { photoelectrons in each } \\
& \int_{0}^{\lambda} \mathrm{N}_{\mathrm{p} . e .} \mathrm{d} \lambda_{\mathrm{s}} \simeq 200 \text { photoelectrons }
\end{aligned}
$$

in each spatial element. So if one assumes that the amplification process is noiseless and that external noise can be neglected, the signal-to-noise ratio of the density estimate is $\left[200 \mathrm{~N}_{\mathrm{e}}\left(\mathrm{cm}^{-3}\right) / 10^{13}\right] 1 / 2$ and the estimate of $T_{e}$ from a least squares fit will have a similar accuracy. Our detection system will have to come very close to this ideal performance to be useful.

In addition to sensitivity there are several other requirements of the detector. The dynamic range necessary to make the measurements needs to be at least $10^{3}$. The detector must not be saturated by the plasma light which gets through the shutter and is integrated for about 1s. The resolution must be at least as good as that of the spectrometer $(\sim 3 \mathrm{l} / \mathrm{mm}$ referred to the photocathode). For rapid calibration and analysis the device should be linear.

Our detector consists of a $40 \mathrm{~mm}$ diameter MCP image intensifier (Galileo Electro-óptics Córp. - yo40-1131) fiber optically coupled to a $25 \mathrm{~mm}$ diameter SIT television tube. (RCA - 4826). The SIT (12) tube by itself is not quantum noise limited nor is the shutter ratio high enough for our experiment. We have supplemented it with a gated MCP intensifier which has sufficient gain to allow us to work near the top of the SIT dynamic range. In order to see what the 
limitations are we note that the limiting noise in the SIT tube is switching noise from the electron reading beam (13). The noise charge is $: Q_{N}=\left(\left.2 \mathrm{kT}_{\mathrm{b}} \mathrm{c}_{\mathrm{p}}\right|^{1 / 2}\right.$ where $\mathrm{T}_{\mathrm{b}}$ is the temperature of the beam $\left(\sim 1100^{\circ} \mathrm{k}\right.$ ) and $\mathrm{C}_{\mathrm{p}}$ is the capacity of one pixel. For our pixels referred to the $18 \mathrm{~mm}$ diameter target $Q_{N} / e \simeq 10^{3}$ electrons. The upper end of the range can be estimated from the diode array bias voltage. This is recommended to be $8 \mathrm{~V}$ so if one takes saturation at $430 \%$ of this value, the saturation charge per pixel is $Q_{\mathrm{s}} / \mathrm{e} \simeq 0.3 \mathrm{C}_{\mathrm{p}} \mathrm{V}_{\mathrm{b}} / \mathrm{e} \simeq 1.5 \times 10^{7}$. electrons. Thus the dynamic range can be $1.5 \times 10^{4}$ provided dark current doesn't saturate the target in a frame. In fact the observed noise is about 10 times the switching noise limit. The excess noise is predominately acoustical pickup from vibrations in the target membrane and while it can be subtracted out electrically by chopping the read beam we have taken the luxury of using only the upper end of the rarige.

The current gain in the SIT tube is about 2000 , so it would appear that a gain of only 10 would be sufficient to let us resolve one photo-electron; however the loss in the reducing fiber optic coupling element is about 10, so we actually need a gain of at least 100 in the intensifier.

The MCP intensifier was originally choosen because of its gating: speed (14) and high shutter ratio (ratio of signal when the gate is open to that when it is closed). Secondarily the tube can be made with a flat glass window which can be efficiently matched to our $\mathrm{f} / 1$ optics. The tube used here has a proximity focused front and back end, an extended red s-20 photcathode, and a P-20 phosphor output. The photocathode is prepared on a transparent conducting substrate; otherwise the resistivity of this type of 
photocathode is too high to allow the 100 ns gate to charge it uniformly. In our devise the gate is $90 \%$ open across the entire face within $10 \mathrm{~ns}$. The voltages across the MCP and between the MCP and the phosphor are left on as in its more usual D.C. operation. Saturation effects can be estimated for the MCP intensifier from some of its physical and electrical characteristics. Normally the MCP secondary emitting surface is made tor D.C. operation, so the channel resistance is high $\left(\sim 500 \mathrm{M} \Omega-\mathrm{cm}^{2}\right)$. The channel length is about $0.5 \mathrm{~mm}$ so that the RC time of the plate is $\sim 10^{-3} \mathrm{~s}$. Thus the channels cannot recharge during our 100 ns signal window and the average number of photo-electrons per channe 1 must be kept below one to insure a linear response. We estimate $10^{4} \mathrm{channels} / \mathrm{mm}^{2}$ or $2 \times 10^{3}$ channels/pixel. For the signal estimates given previously saturation can be avoided as long as $\mathrm{N}_{\mathrm{e}} / \mathrm{T}_{\mathrm{e}}^{\mathrm{l} / 2}<5 \times 10^{14} \mathrm{~cm}^{-3}$ / $\mathrm{kev}^{1 / 2}$.

Both the MCP intensifier and the SIT vidicon are electrically gated. The MCP photocathode is biased $+30 \mathrm{~V}$. with respect to the MCP input and a $-300 \mathrm{~V}$ gate pulse is applied to turn it on. The shutter ratio of the intensifier alone is about $10^{5}$. The response of the phosphor to the short pulse is nonlinear decaying slowly in time. We gate $95 \%$ of the phosphor light through the SIT tube with a 200 us sate applied to the focus/gate electrode. The SIT tube is normally biased off by holding the focus/gate electrode at $-11 \mathrm{kv}$, while the photocathode is at $-10 \mathrm{kv}$. Thus most of the MCP intensifier output is integrated on the SIT target which is 
cooled to $-35^{\circ} \mathrm{C}$. The shutter ratio of the combination is about $10^{8}$.

The intricacies of obtaining a linear response from cooled silicon vidicons has been dealt with by others $(3,4,5)$. The readout is inefficient for signal voltages less that $\mathrm{kT}_{\mathrm{b}}$ leading to an apparent nonlinearity in the response of the tube for signals less than $\sim 1 / 30$ the saturation level. While the nonlinearity could be corrected after the readout, the signal-to-noise ratio quickly becomes limited by amplifier and internal noise at low signal levels. To avoid the nonlinearity the target potential is raised slightly between erasure and readout so that the beam always lands with an energy of about $4 \mathrm{kT}_{\mathrm{b}}$. The dark field then appears as a pedestal about $15 \%$ of full scale. After the target is read out and the potential returned to its normal level however the beam will not land. To restore the target to a consistent initial state some leakage current must be introduced. This may be done by saturating the diodes with a flash of light or by drasticaliy altering the target potential. We have had good results by following the procedure recommended by $R C A$ for removing charge stored on the dielectric between diodes. The main feature of this erase process consists of raising the target to $+300 \mathrm{~V}$ and increasing the beam current to its maximum. The entire erase cycle is shown in Table 1. The linearity and $\mathrm{S} / \mathrm{N}$ for a single pixel, for the MCP-SIT combination, arid for the range $1 \hat{\imath}_{S} / \mathrm{N}^{<} 20$ are shown in figure 7 . The measured range is about 500:1.

The MTF referred to the MCP photocathode is $50 \%$ at $3 \mathrm{l} / \mathrm{mm}$. The response of the system to a flat input field is markedly 
nonuniform falling off by as much as a factor of two at the edges. This is due to the combined effects of the MCP, fiber optic reducer, and SIT beam landing; however the $S / N$ ratio is uniform to $20 \%$ and the system is linear over the entire field. The experimental configuration is shown is figure 8 . The entire camera is enclosed in an annealed soft iron box $6.3 \mathrm{~mm}$ thick to shield the SIT electron optics from the ambient fields. Secondary shielding is provided by a double wall of $1.6 \mathrm{~mm}$ high permeability alloy. The box is insulated but only the target flange is actively cooled. Acetone at $-35^{\circ} \mathrm{C}$ circulates through a copper plate which makes a thermal contact with the SIT flange. The remaining components cool by conduction, the MCP reaching about $0^{\circ} \mathrm{C}$ in equilibrium. The apparatus is assembled by loading the MCP-SIT assembly from the rear of the iron box. The camera is spring loaded, so that the MCP face plate is pressed against a window which separates the cooled enclosure from the camera lens and which serves as a reference plane for the focius.

The total number of pixels on the image field is $20 \times 80=1600$ (counting the four blank lines which separate the scattered and plasma background channels). In order to limit the data storage to this number we scan the target in a nonstandard way. Figure 6 shows a small portion of the read beam pattern. The beam sweeps 80 horizontal lines driven by a single sawtooth but the vertical sweep has a fine sawtooth superimposed on it. The beam width is 
adjusted so that the entire area is swept out one pixel after another. The current from each pixel is integrated, digitized (10 bit), and stored successively in the memory of a PDP-11/40 computer. The fine sawtooth is the fundamental clock of the system operating at $62.7 \mathrm{kHz}$. There are 16 cycles/pixel, 20 pixels/ line, and 80 lines altogether making one frame 0.43s. long(counting 80 line return pixels and one return line). The horizontal and vertical deflection colls are both designed (Celco B2741-1) to reduce cross coupling at high frequencies.

The organization of the camera electronics is shown in figure 9. The system is designed to operate either as an independent camera - for alignment - or under the control of a sequencer-which steps the camera through an erase, expose, read cycle - or as a real time data processor - which acquires and processes raw data to get $\mathrm{N}_{e}$ and $\mathrm{T}_{e}$ profiles synchronous with PLI operations. Since the camera must be run remotely, the electronics are split into two parts. The sweep generators, preamplifier and video processing, and the high voltage power supplies and gates are near the spectrometer. The SIT biasing, camera controlling and sequencing, and digital processing are all in the control room., The initial set up of the camera can be done with the video monitor. The array size and number of sawteeth per pixel are set on a switch register in the sync generator. Then the horizontal and vertical sweeps are adjusted so that the scan array matches the spectrometer pattern. Having aligned the camera, the sequencer can step through an erase, expose, read cycle for a close look at the single exposure mode of operation. 
Finally fully automatic operation requires the laser to be charged and target erased prior to a plasma pulse. The laser and shutter are fired synchronously at a preset time in the discharge, and the target is readout after a fixed delay from the end of the erase cycle. The frame is digitized and transferred to a PDP-10 for analysis along with other PLT diagnostic data.

Optical Alignment

The alignment of the laser relative to the spectrometer needs to be done accurately and remain so during the plasma pulse. The laser and beam deflector rest on the concrete basement floor which in turn rests on bed rock; so the laser beam path is a good stationary reference. The spectrometer rests on the general diagnostic platform. This floor is shored up by 10 " aluminium I beams and is mechanically independent of the PLT support structure. Movements in the floor are caused by eddy currents induced in the support lattice; however the image of the spectrometer slit is observed to move less than $0.2 \mathrm{~mm}$. The slit image is sufficiently wide to absorb the error.

The laser and fiber optic dissector are aligned with the aid of a small screen which can be rotated into the beam and slid along it (partly visible in its retracted position in the top of figure 3). A HeNe laser is adjusted to fill the beam optics in the same was as the ruby laser. We then adjust the position of the spectrometer by observing the HeNe beam and the illuminated image of the optic fiber bundles on the screen. The apparatus has been run for several months without readjustment. 


\section{Calibration}

We have used two methods to calibrate the apparatus. The simplest and the one which we normally use as a standard consists of a uniformly illuminated white screen. The screen is large enough to fill the aperture of the objective lens. It is coated with a uniformly reflecting barium sulfate composition (Eastman White Reflectance standard) and illuminated with a tungsten halogen lamp. The absolute spectral intensity is calibrated against. an NBS standard tungsten ribbon lamp.

The number of scattered protons per unit wavelength interval is

$$
\mathrm{N}_{s}\left(\lambda_{s}, \theta_{s}\right) / \Delta \lambda=N_{i} N_{e}\left(\theta_{s} j r_{o}^{2} L\left(\theta_{s}\right) \Delta \Omega\left(\theta_{s}\right) F\left(\lambda_{s}, \theta_{s}\right) \text { photons } / \AA\right.
$$

where $F\left(\lambda_{s}, \theta_{s}\right)$ is a normalized shape factor $\int_{-\Delta \Delta}^{00} F\left(\lambda_{s}, \theta_{s}\right) d \lambda_{s}=I$. lhe white screen distribution is

$$
N_{\omega}\left(\lambda_{s}, \theta_{s}\right) / \Delta \lambda=I\left(\lambda_{s}\right) A\left(\theta_{s}\right) \Delta \Omega\left(\theta_{s}\right) \sin \theta_{s} \cdot T \text { photons } / \AA
$$

where $I\left(\lambda_{s}\right)$ is the calibrated intensity (photons/s-cm ${ }^{2}-s t e r-\AA$ ), $A\left(\theta_{5}\right)=L\left(\theta_{6}\right) W\left(\theta_{6}\right)$ is the area of a light pipe element.projected on the laser beam, and $\mathrm{T}$ is the gate width. The ratio of the scattered signal $S_{S}$ to the white screen signal $\left(S_{\omega}\right)$ give a set of sensitivity factors $R\left(\lambda_{S}, \theta_{S}\right)$ for each pixel. We have 


$$
\frac{\mathrm{S}_{\mathbf{s}}}{\mathrm{S}_{\omega}}=\frac{\mathrm{N}_{\mathbf{s}}}{\mathrm{N}_{\omega}}
$$

and

$$
\mathrm{N}_{\mathrm{e}}\left(\theta_{\mathrm{s}}\right) \mathrm{F}\left(\lambda_{\mathrm{s}}, \theta_{\mathrm{s}}\right)=\frac{\mathrm{s}_{\mathrm{s}}\left(\lambda_{\mathrm{s}}, \theta_{\mathrm{s}}\right)}{\mathrm{R}\left(\lambda_{\mathrm{s}}, \theta_{\mathrm{s}}\right)}
$$

where

$$
R\left(\lambda_{s}, \theta_{s}\right)=\frac{s_{\omega} N_{i} r^{2}}{I\left(\lambda_{s}\right) w\left(\theta_{s}\right) T \sin \theta_{s}}
$$

The width factor $W\left(\theta_{s}\right)$ depends on objective lens design. For our concentric system it is simply

$$
W\left(\theta_{s}\right)=\frac{W(\pi / 2)}{M_{0}}\left(\frac{1+M_{0}}{\sin \theta_{s}}-1\right)
$$

where $M_{O}$ is the axial magnification $\left(M_{0}=1 / 0.53\right)$ and $W(T / 2)=0.3 \mathrm{~cm}$.

Calibration by this procedure has several inherent difficulties. First the source is on continuously and the actual signal is pulsed. If the response of the detector is the same across the $100 \mathrm{~ns}$ gate width and if the gate has the same width for all pixel elements, then the pulsed and continuous source response should be the same. Detailed measurements of the gate shape show that the temporal and areal variations should cause less than 5\% errors in the calibration. This uncertainity can cause systemmatic errors in both the temperature and density estimates. Another class of difficultiès affects mainly the transmission as a function of position (or $\theta_{s}$ ) and therefore only disturbs the shape of the density profile. The most obvious of these errors would be a misalignment of the spectrometer with respect to the laser beam. However there is also 
the possibility of vignetting in the vacuum chamber window or of small variations in transmission within each fiber bundle. We estimate that systemmatic errors from all of these sources can lead to errors of $5 \%$ in $\mathrm{T}_{e}$ and $15 \%$ relative error in $\mathrm{N}_{\mathrm{e}}(\mathrm{r})$. No serious attempt is made here to absolutely calibrate the density by this method. We infer a shape and set the scale to match the line density $\left(\int \mathrm{N}_{e} \mathrm{~d} l\right)$ as measured over a similar vertical chord by microwave interferometry.

The second calibration method is Rayleigh scattering. The vacuum vessel is filled with the current working gas $\left(\mathrm{H}_{2}, \mathrm{D}_{2}\right.$, He etc.) to avoid contaminating the vessel walls with impurities. The ruby laser is scattered from the neutral gas in the same way as from the plasma except that the grating must be rotated slightly to allow the scattered signal, "which for our purposes is unshifted, to fall on the image field of the camera. since the scattering source geometry and cross section are identical except for a known constant to the scattering from a uniformly dense plasma, most of the uncertainty of the previous method may be eliminated. We use this technique to calibrate a single wavelength channel near the line center. Differences between the white screen and Rayleigh calibration can be introduced as a set of fudge factors in the sensitivity array generated by the former method.

When the vessel is evacuated we observe a stray light level equivalent to scattering from a free electron density of $2 \times 10^{12} \mathrm{~cm}^{-3}$ ( 0.1 Torr $\mathrm{H}_{2}$ ) on the central half of the diameter. On the bottom 
and top the stray light saturates the detector if no attentuation is introduced. Scattering from 20 Torr of $\mathbf{N}_{2}$ with a $x 100$ attentuator is compared with the white screen calibration in figure 10. The ratio of the Rayleigh scattered intensity to the white screen intensity (with gometrical factors taken out) is shown versus radius. The deviation from a straight line must represent variations in the transmission across individual fiber bundles. Small changes in the laser focusing and divergence can change the pattern greatly. Data Analysis

A number of different data files must be collected before we begin to calculate temperatures and densities. The sensitivity and noise in each pixel are found by taking repeated exposures of the white screen. The sensitivity is calculated from the average response and the standard deviation is used to assign a noise to subsequent single exposure signals scaling the noise as (signal) $1 / 2$. While the tokamak is running we take four kinds of data corresponding to the four possibilities for firing the laser and plasma (both, either, or neither). When the laser and plasma are on at the same time we get a signal which consists of scattered + plasma + stray + zero level light. The stray laser light is determined by firing 
the laser slightly before the initiation of the plasma pulse. The zero levels are found similarly. And the plasma light can be estimated either by leaving the laser off on one pulse and observing the light on the signal channels or by inferring the plasma light from the "background" channels. When the plasma light is reproduceable this allows us to check our single shot method of estimating the plasma light. All data are stored in disk files which can be manipulated between shots. The most current data files can be plotted for a given position element on a display terminal (see figure 11).

The analysis consists of least squares fitting an exponential curve through the observed scattered signals. Including order $\mathrm{v} / \mathrm{C}$ relativistic effects $(15,16)$ the function to be fitted is

$$
F\left(\lambda^{\prime}\right)=\frac{\left(1-5 / 2 \lambda^{\prime}\right)}{\pi^{\frac{1}{2}} \lambda_{i} \delta} \quad e^{-\frac{\lambda^{\prime 2}}{\Delta^{2}\left(1+\lambda^{\prime}\right)}}
$$

where $\lambda^{\prime}=\left(\lambda_{s}-\lambda_{i}\right) / \lambda_{i} \leq 0.2$. We minimize the function

$$
\left.x^{2}=\sum_{j=1}^{20}\left[I_{j}-A\left(1-5 / 2 \lambda_{j}^{\prime}\right) e^{s} \frac{\lambda_{j}^{2}}{1+\lambda_{j}^{\prime}}\right]\right]_{\sigma_{j}^{2}}^{2}
$$


where the sum is over each wavelength channel, $I_{j}, \sigma_{j}$ are the data to be fitted and its standard deviation, $A=k n_{e} / \lambda_{i} \delta$, $s=1 / \delta^{2}$ and $k$ is to be determined from interferometric measurements of $\int \mathrm{N}_{\mathrm{e}} \mathrm{dl}$. An initial guess is made by reducing the problem to a weighted linear least squares fit to $\ln I_{j}=\ln A+s \lambda^{\prime} j^{\prime} x^{2}$ is then expanded about the initial guess $A_{0}, S_{0}$ and an increment $\Delta \mathrm{A}, \Delta \mathrm{S}$ is found by reapplying the linear least squares formulae. Usually 4 or less iterations are necessary to obtain a percent accuracy in $A$ and $s$ which minimixe $x^{2}$. Errors in the values of $A$ and $S$ are found from

$$
\begin{aligned}
\sigma_{S}^{2} & =\sum\left(\frac{\partial S}{\partial I_{j}}\right)^{2} \sigma_{j}^{2} \\
\sigma_{A}^{2} & =\sum\left(\frac{\partial A}{\partial I_{j}}\right)^{2} \sigma_{j}^{2} \\
\sigma_{A}^{2}, S & =\sum\left(\frac{\partial A}{\partial I_{j}}\right)\left(\frac{\partial S}{\partial I_{j}}\right)_{j}^{2}
\end{aligned}
$$

where the partials are found analytically by implicit differentiation of the minimization conditions.

$$
\frac{\partial x^{2}}{\partial A}=0, \frac{\partial \chi^{2}}{\partial S}=0
$$


Finally we obtain

$$
\begin{aligned}
\sigma_{T_{e}}{ }^{2} & =\sigma_{S}^{2}\left(T_{e} / S\right)^{2} \\
\underset{\sigma_{k N}}{2} & =\left(k N_{e}\right)^{2}\left[\sigma_{s}^{2}(1 / 2 S)^{2}+\sigma_{A}^{2}(1 / A)-\sigma_{A, S}{ }^{2}(1 / A S)\right] \\
\sigma_{T_{e}, k N_{e}} & =N_{e} T_{e}\left[\sigma_{s}{ }^{2}\left(1 / 2 S^{2}\right)-\sigma_{A, S}{ }^{2}(1 / A S)\right]
\end{aligned}
$$

A typical fit is shown in "figure 12 and temperature and density profiles are shown in figure 13 for a relatively high density helium discharge. The noise in the density estimate is about twice as large as we would have predicted based on the efficiency of our optics and camera assuming no noise other than that from shot noise from the scattered photons. We attribute the discrepancy to noise from plasma light, and from the laser intensity monitor all of which are included in the error estimate calculated from the data. At high densities systomatic erroro of undetermined origin become important. This is indirated by a

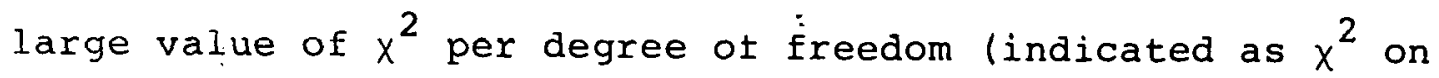
figure 12). Nevertheless a number of interesting features can be seen in the $\mathrm{T}_{\mathrm{e}}$ profile. The small hollow region in the center is probably evidence of a thermal instability brought on by impurity radiation in the center. Small steps can also be seen at integral $q$ radii (arrows). The error observed over many sets of data may be summarized in the following formula.

$$
\frac{\Delta N}{N_{e}}=\frac{b}{2} \frac{\Delta T_{e}}{T_{e}}=0.3\left[\therefore 0^{13} / \mathrm{N}_{e}\left(\mathrm{~cm}^{-3}\right)\right]^{1 / 2}
$$

Conclusion

Our multichannel Thomson scattering apparatus has been collecting data since July 1976. 'It has operated reliably producing data which for a single position element are somewhat inferior 
to other "single position" instruments. The reasons for this are clear: 1) the multichannel instrument must use a transparent photocathode and is therefore restricted to multialkali photocathodes whose quantum efficiency is 5-10\% in our spectral range. Instruments which employ photomultipliers may use the higher quantum efficiency (20-30\%) Gallium Arsenide reflecting photocathodes. 2) A relatively inefficient image dissector must be used to match the laser beam to the photocathode. 3) The size of suitable detectors ultimately limits the collection solid angle in this case more severely than in a single position instrument. Nevertheless the time required to produce profiles of comparable accuracy has been considerably reduced.

When plasma discharges are irreproducable the multichannel device has a singular advantage. For example we have clearly identified hollow temperature profiles even when such discharges occur randomly mixed and otherwise indistinguishable from normal ones. We hope in the future to look at profile changes which may occur durıng disruptions associated with low limiter q values or the high density limit.

Acknowledgament

Many people have contributed to this project in the three years of development. Paul Zucchino and John Lowrance of the Princeton University observatory shared their considerable experience with astronomical sensors and provided technical assistance in building the electronics system. Karen Hill and 
Wayne Roney have been responsible for most of the software and Joe Mayercak has kept the computer interface intact through innumerable changes. Finally, Ken Hobson, Dick Holzbaur, and Andy Siroki have assembled most of the mechanical and electrical jungle left mercifully vague in the preceding paper.

* Work supported by U.S. E.R.D.A. Contract EY-76-C-02-3073

+ Princeton University Observatory 
References

1. Desilva, A. W., Goldenbaum, G. C., in Methods of Experimental Physics, H. R. Griem and R. H. Losberg, Eds., Academic Press, New York, N.Y., 1970, Vol. 9A, 213.

2. Dimock, D. L., Eubank, H. P., Hinnov, E., Johnson, L. C., Meservey, E. B., Nucl. Fus. 13 (1973) 271.

3. McCord, T. B., Westphal, J. A., Appl. Opt. 11 (1972) 522 .

4. Colgate, S. A., Moore, E. P., Colburn, J., Appl. Opt. 14 (1975) 1429 .

5. Hunten, D. M., Stump, C. J., Jr., Appl. Opt. 15 (1976) 3105 .

6. Burns, F. P., IEEE Spectrum 4 (1967) 115.

7. Private Communications with W. J. Rundle of Korad with respect to a low divergence laser system built for the Lunar Ranging Experiment.

8. Winer, I. M., Appl. Opt. $\underline{5}$ (1966) 1437.

9. Designed by A. McGill and J. Hoagland of Fairchild Camera.

10. James, J. F., Sternberg, R. S., The nesign of optical Spectrometers, Chapman and Hall Ltd., London (1969) 51.

11. Bekefi, G., Radiation Process in plasmas, J. Wiley and Sons, New York, N.Y. (1966).

12. Singer, B., IEEE Trans. on El. Dev. ED-18 (1971) 1015.

13. Beurle, R. L., Proc. IEEE 110 (1963) 1350.

14. Lieber, A. J., Rev. Sci. Inst. 43 (1972) 104.

15. Pechacek, R. E., Trivelpiece, A. W., Phys. Fluids 10 (1967) 1688. 16. Sheffield, J., Plas. Phys. 14 (1972) 783. 
Table I.

\begin{tabular}{|c|c|c|c|}
\hline FRAME & BEAM & SCAN SIZE & TARGET \\
\hline $0-21$ & HI & OVER & HI \\
$22-32$ & OFF & OVER & NORMAL \\
$33-40$ & ON & OVER & NORMAL \\
$41-64$ & ON & NORMAL & NORMAL
\end{tabular}

The target erasure consists of 64 frames. The first are done with the target potential raised to $300 \mathrm{v}$ and the beam current at its maximum value. Subsequent frames erase the target with normal target potential. 




773166

Fig. 1. The physical layout of the laser, plasma, and spectrometer are shown. 


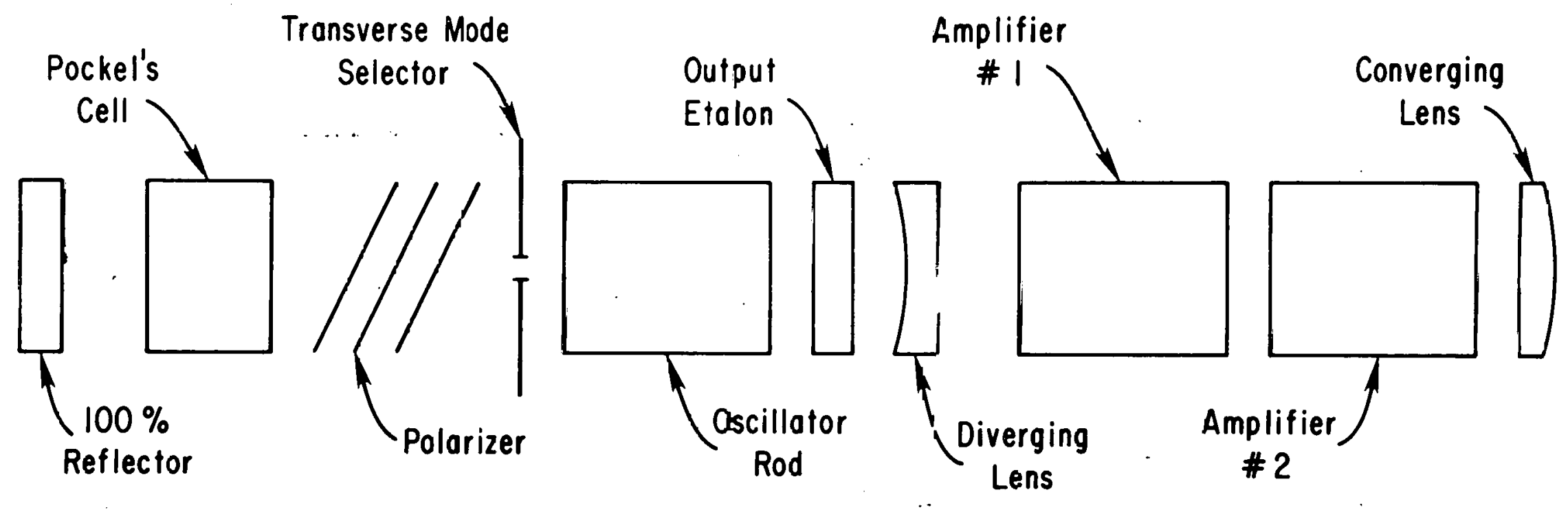

Fig. 2. The low divergence laser consists of an aperture constrained oscillator and Pockel's cell followed by two amplifiers. 




766253

Fig. 3. A wide angle photograph shows the razor blade stack viewing dump. Several of the beam entrance and exit apertures can be seen. 


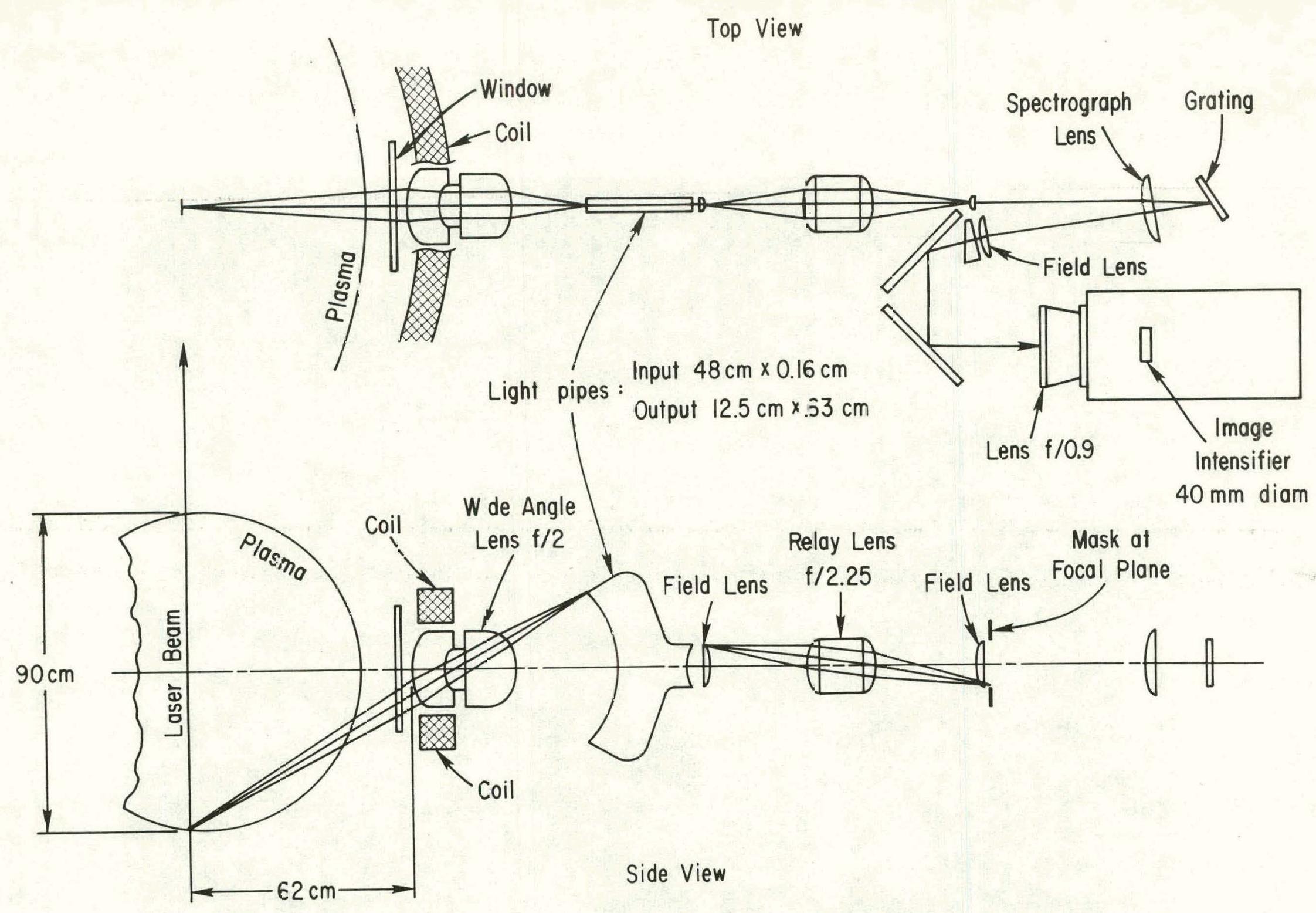

Fig. 4. A top and side view show the ccmponents of the light collection optics, spectrometer, and TV camera. 


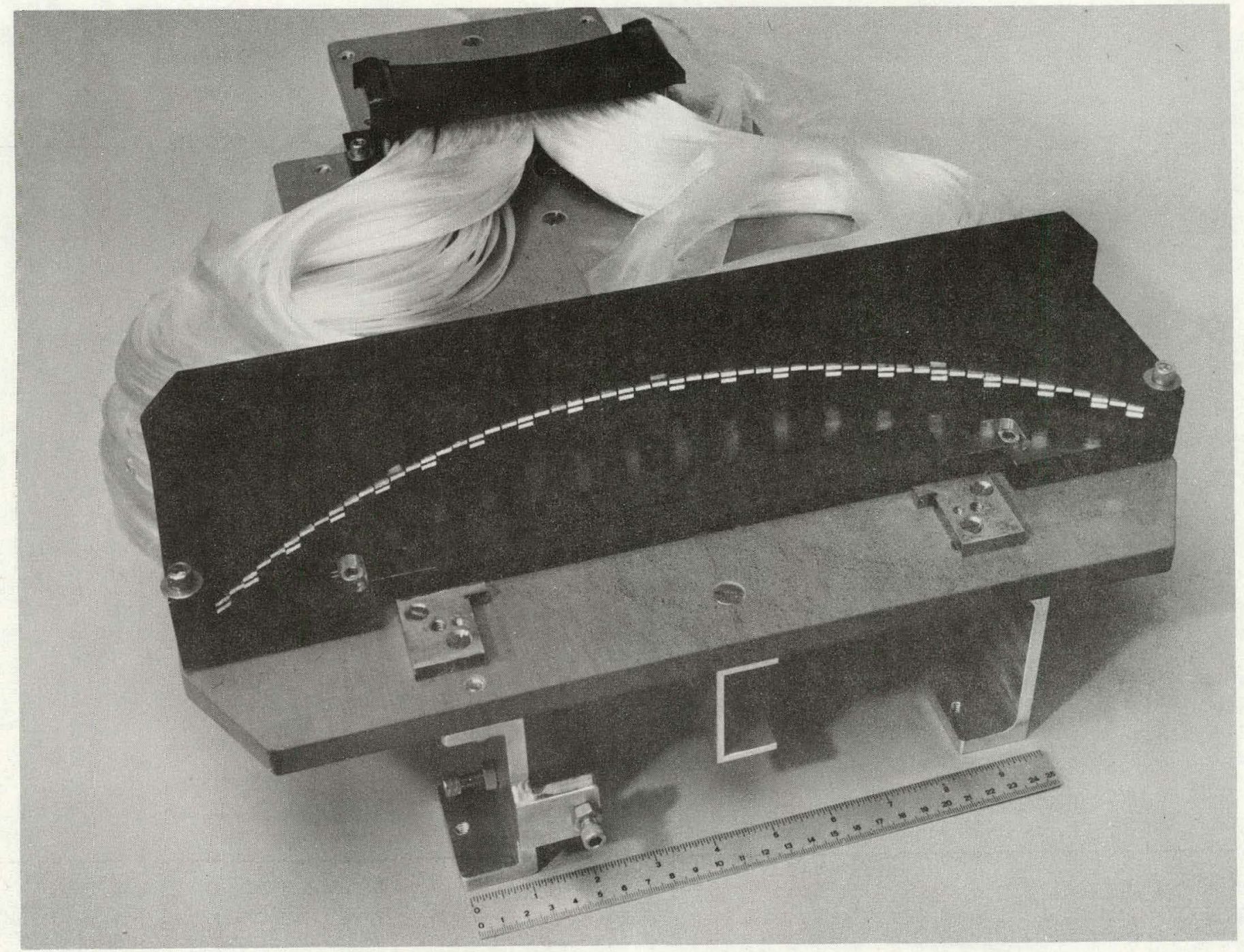

763250

Fig. 5. A photograph shows the objective image surface of the fiber optic image dissector and mount. 


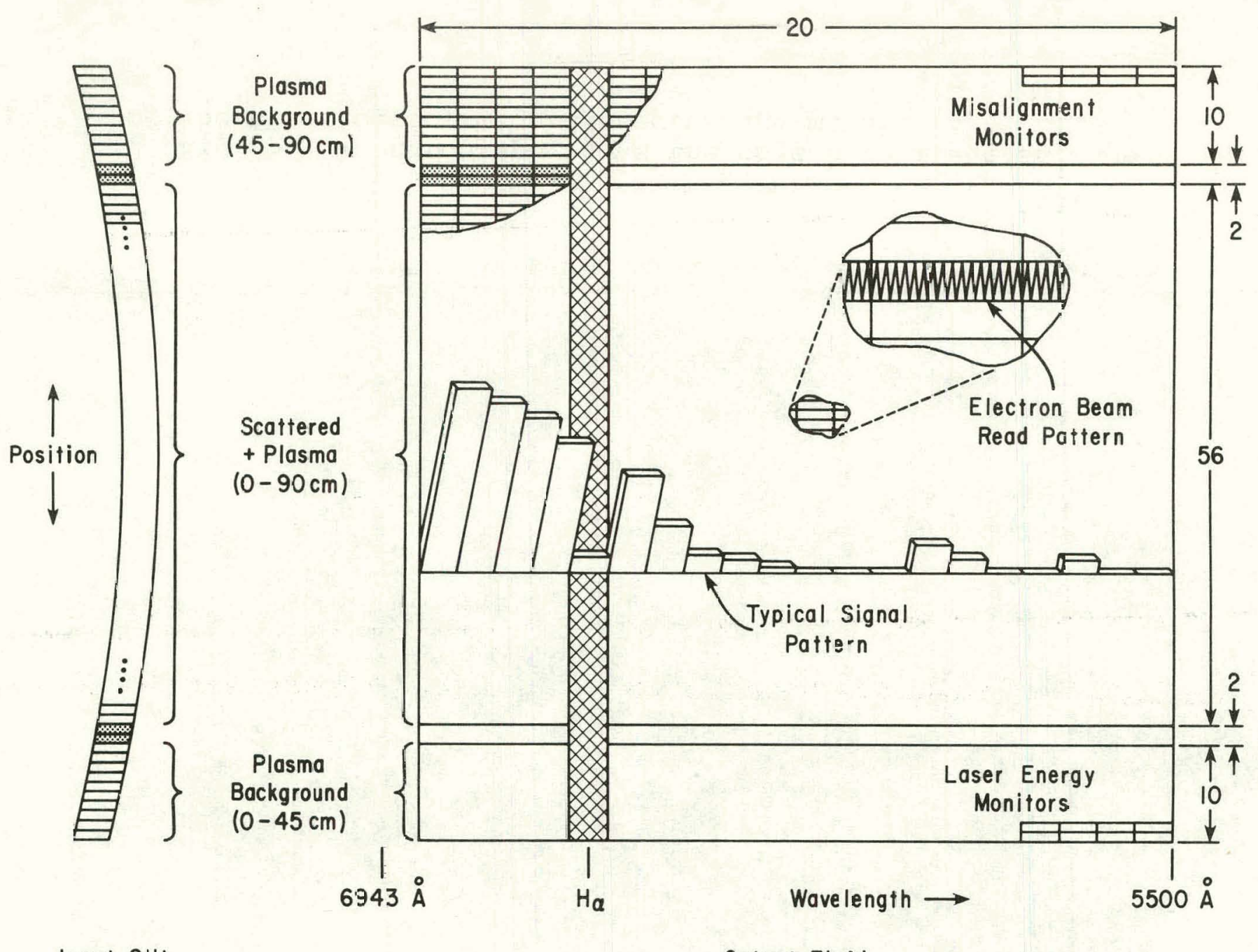

Irput Slit

Iig. 6. The geometry of the spectrometer input slit and output fiela are shown. The scattered light appears on the central part of the field while the plasma baskground light is split-half above and half below. 


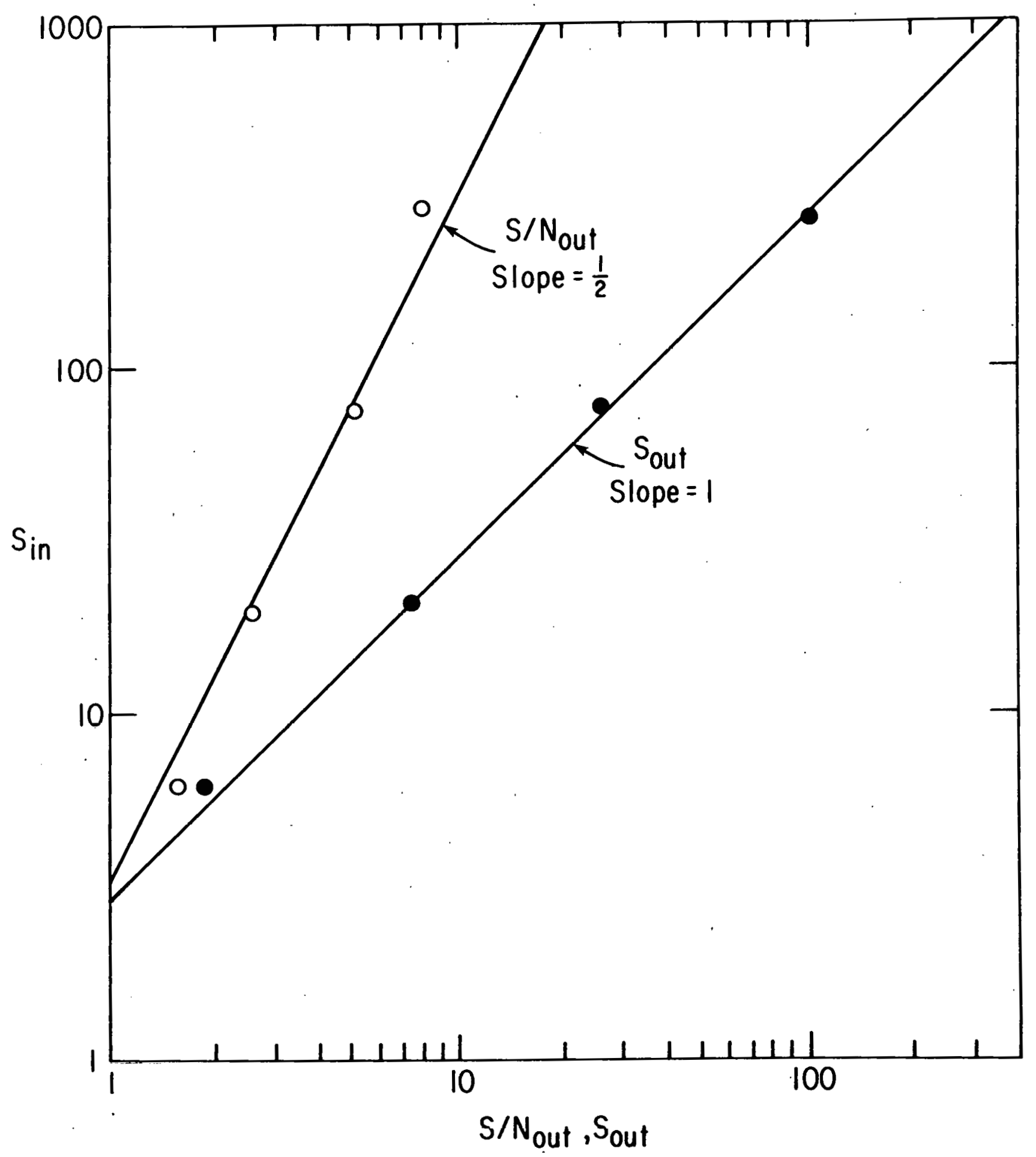

773152

Fig. 7. The response of the MCP-SIT camera is shown to be linear over about 3 dgcades. The signal is integrated over one pixel $\left(\sim 0.1 \mathrm{~mm}^{2}\right.$ referred to the $5 I$ i target). The signal-to-noise ratio scales as shot noise over the range $1 \leq \mathrm{S} / \mathrm{N} \leq 20$. 


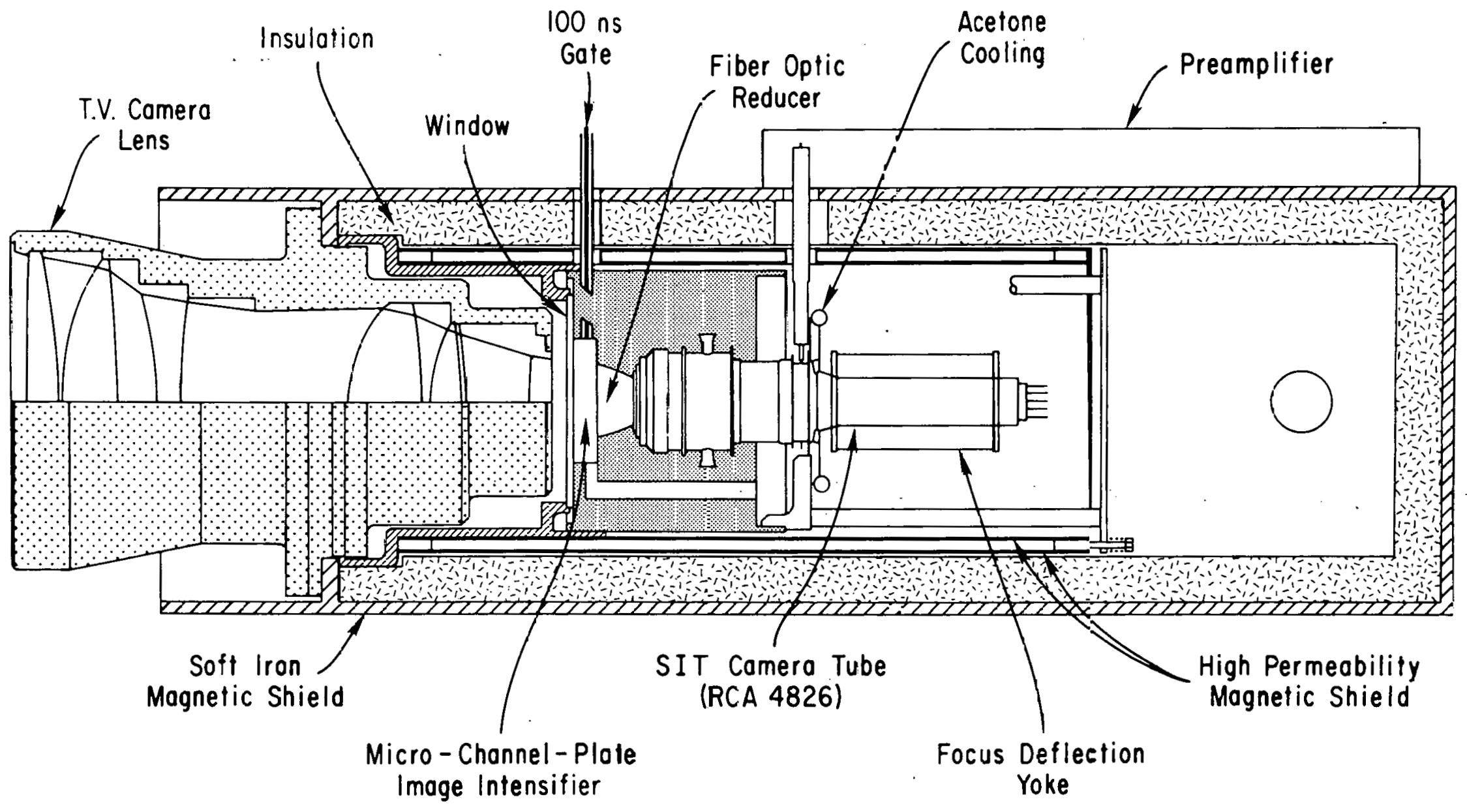

773164

Fig. 8. The TV camera zonsists of an $f / l$ lens and a $40 \mathrm{~mm}$ diameter MCP image intensifier fiber optically coupled to an SIT camera tube. The devize is magnetically shielded and cooled to $-35^{\circ}$ by circulating acetone through a flange in contact with the target. 


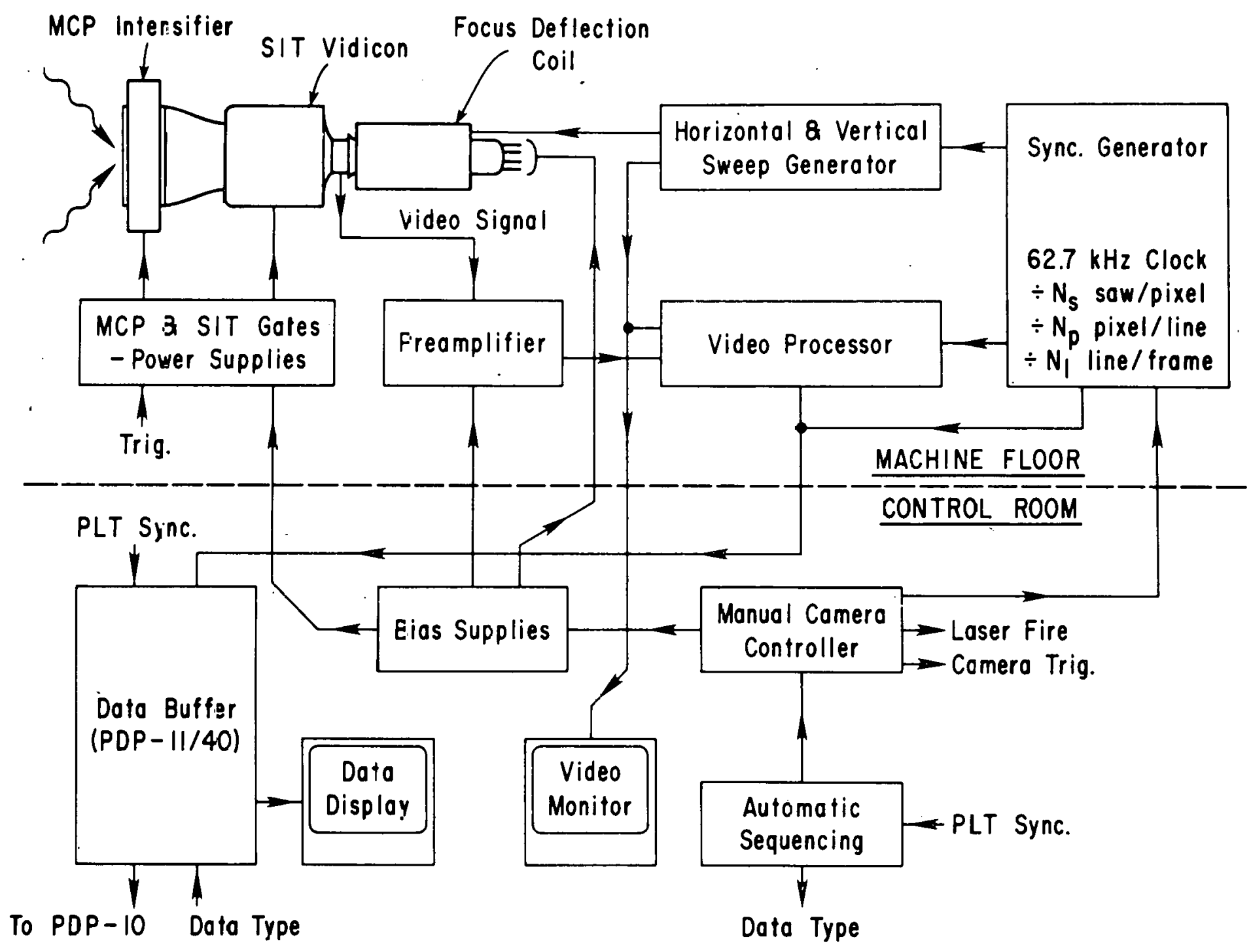
T $\mathrm{V}$ camera electronics. 


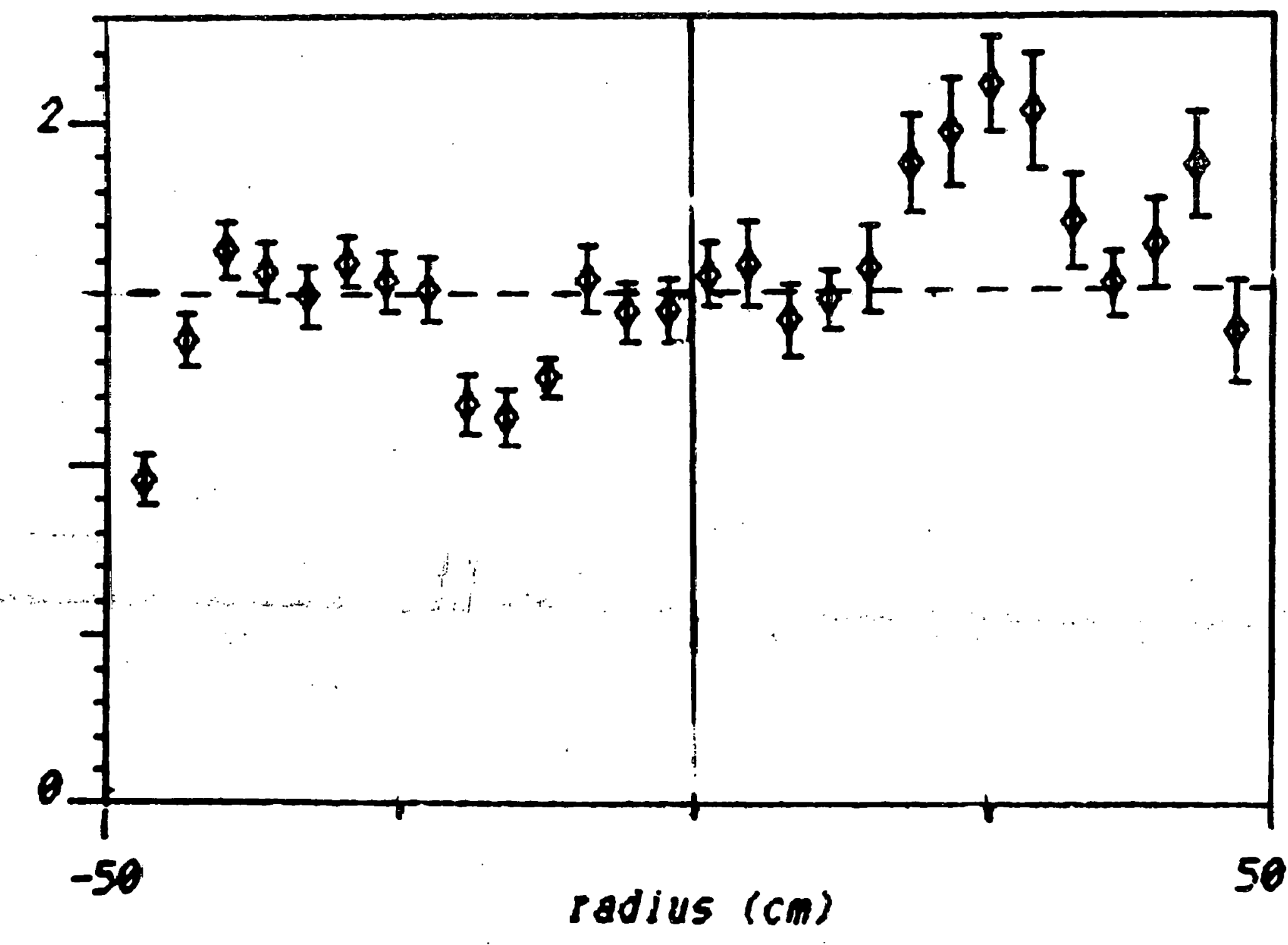

773380

Fig. 10. The ratio of Rayleigh scattered intensity to the white screen intensity (with geometrical factors taken out) is shown versus radius. 


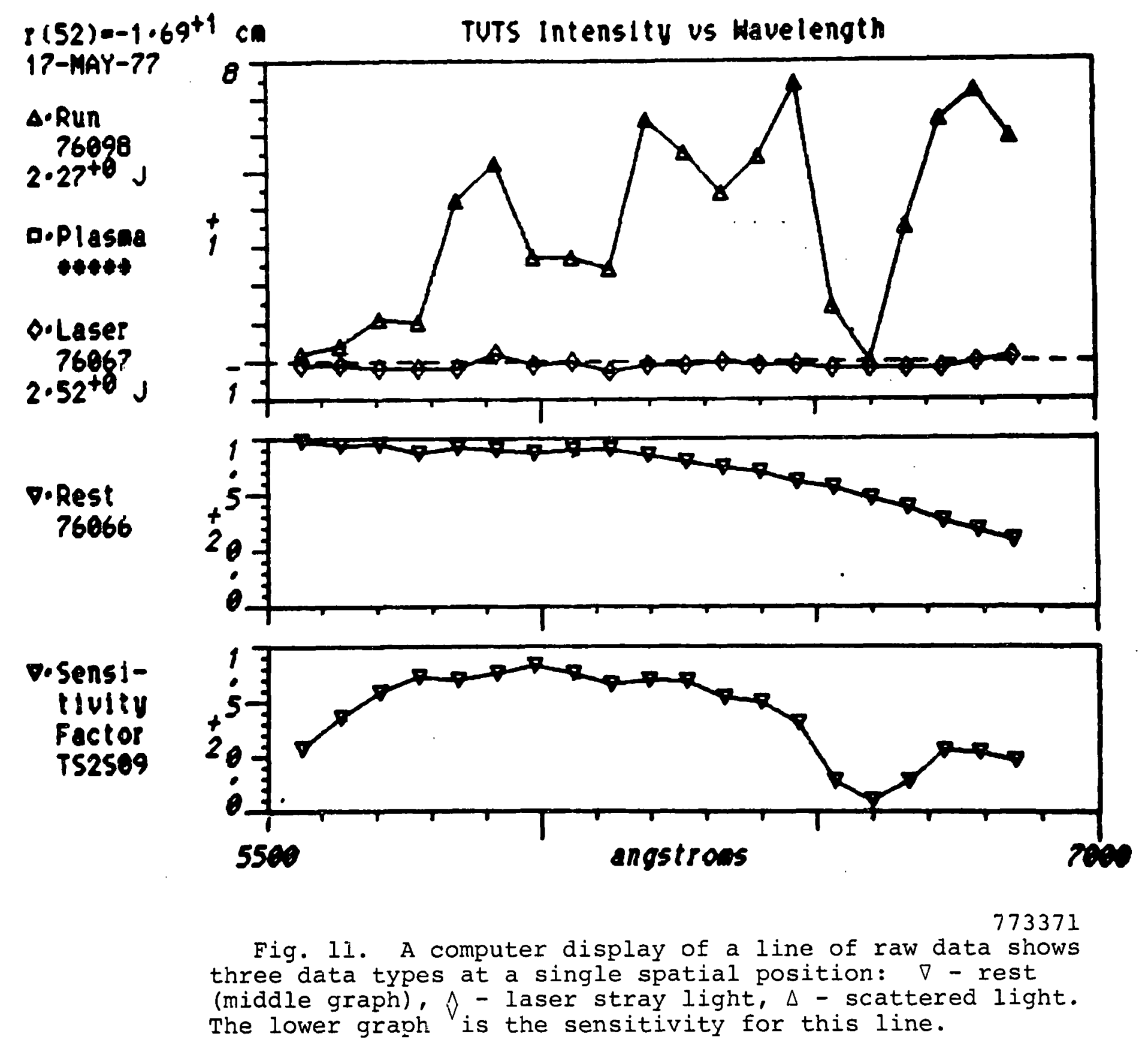
The lower graph is the sensitivity for this line. 
T $0.87 \pm 0.08 \mathrm{keV}$

ก $12.25 \pm 0.4910^{13} \mathrm{~cm}^{-3}$

$x^{2} 9 \cdot 18$
THOMSON SCATTERING

$P(40)-16.9 \mathrm{Ca}$
17-MAY-77

76090

Fit us Havelength

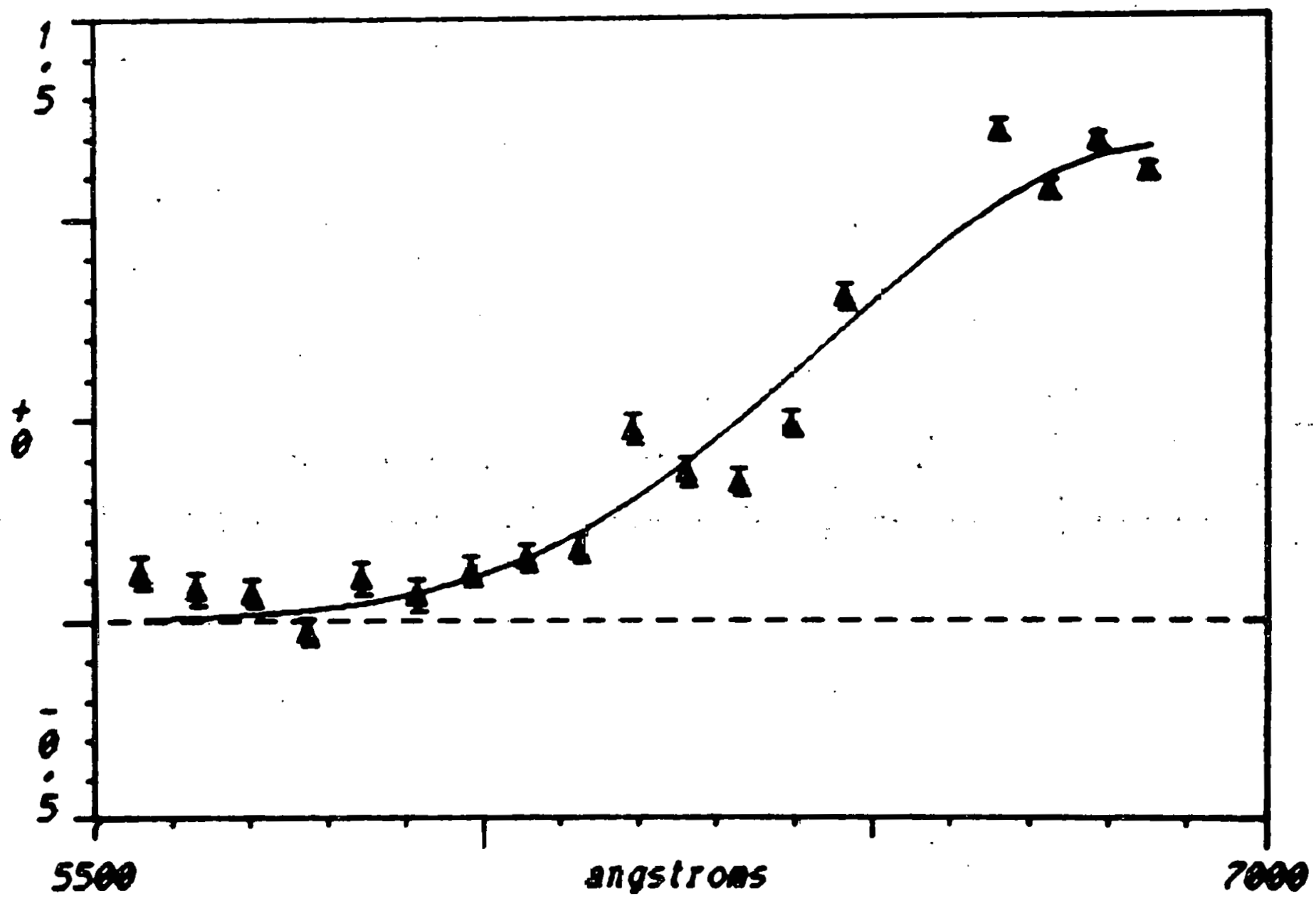

773372

Fig. 12. A relativistically corrected Gaussian curve is fitted tc the blue wing of the scattered profile for

a single position. 


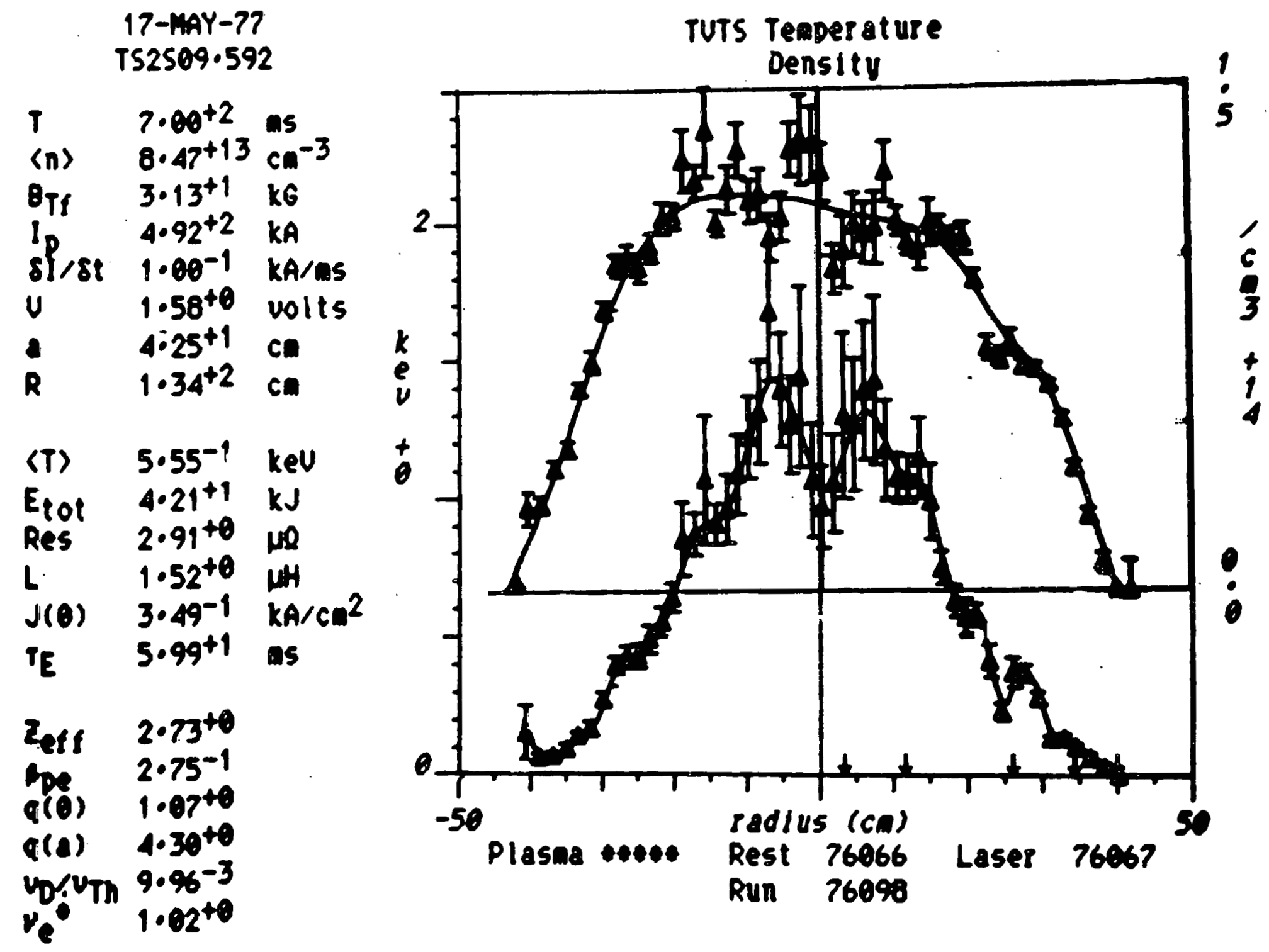

773373

Fig. 13. Electron temperature and density proiiles for a vertical chord through the plasma cross section are shown for a relatively high density He discharge. Various parameters of the discharge and calculations generated from the profiles are displayed on the left (see ref. 2). 